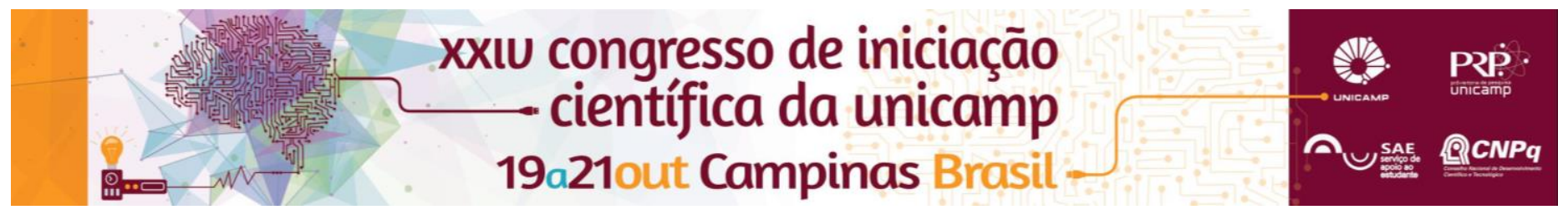

\title{
Análise de algoritmos de reordenação de matrizes baseadas em binarização múltipla
}

\author{
Maressa Rodrigues da Silva*, Celmar Guimarães da Silva
}

\section{Resumo}

A ferramenta Matrix Reordering Analyzer (MRA) efetua experimentos para comparar o desempenho de algoritmos de reordenação de matrizes em termos de tempo de execução e de qualidade das matrizes geradas. Nesse contexto, os objetivos deste trabalho foram: (1) aprimorar a MRA para a realização de testes e experimentos com configuração de parâmetros dos padrões canônicos Simplex, Band e Equi; e (2) adaptar a MRA para a geração automática de gráficos, visando melhor visualização dos resultados e comparações dos algoritmos de reordenação presentes na ferramenta.

\section{Palavras-chave:}

Reordenação de matrizes, Visualização da Informação, heatmaps.

\section{Introdução}

A ferramenta Matrix Reordering Analyzer (MRA) ${ }^{1}$ permite efetuar comparações entre algoritmos de reordenação de matrizes quanto ao tempo gasto nas reordenações e quanto à qualidade das matrizes obtidas como saída dos algoritmos. Nessas comparações, são usadas matrizes sintéticas geradas conforme os padrões canônicos de Wilkinson². Dentre eles, os padrões Simplex, Band e Equi têm parâmetros de configuração que permitem gerar matrizes muito distintas entre si, podendo assim alterar os resultados dos algoritmos de reordenação presentes na ferramenta MRA. Permitir configurar esses valores possibilita ampliar o leque de análises possíveis sobre os resultados desses algoritmos. Assim, esse projeto teve como um de seus objetivos implementar a possibilidade de configurar esses padrões na MRA.

Outro objetivo foi adaptar a ferramenta para gerar gráficos dos experimentos automaticamente, facilitando o processo de análise após a execução de experimentos de comparação.

\section{Resultados e Discussão}

Alterações nos métodos que geravam os padrões foram feitas para que eles se tornassem configuráveis. Exemplos de diferentes configurações do parâmetro "largura de banda" são mostrados na Fig. 1. Além disso, a interface da ferramenta foi alterada para que essas configurações pudessem ser informadas pelo usuário.

\section{Band}
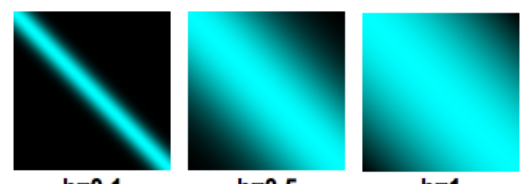

Figura 1. Exemplo de matriz Band com diferentes configurações de largura de banda.

A Fig. 2 exemplifica gráfico estatístico gerado via biblioteca JFreeChart sobre dados de um experimento. Ele informa a média e o desvio padrão de cada resultado da função de avaliação (no caso, stress de Moore) que avalia o resultado dos algoritmos de reordenação.

Parte desta pesquisa possibilitou a aceitação de artigo sobre o algoritmo $\mathrm{SMB}^{3}$, a ser publicado nos anais do congresso internacional IV $2016^{4}$.

DOI: 10.19146/pibic-2016-51601

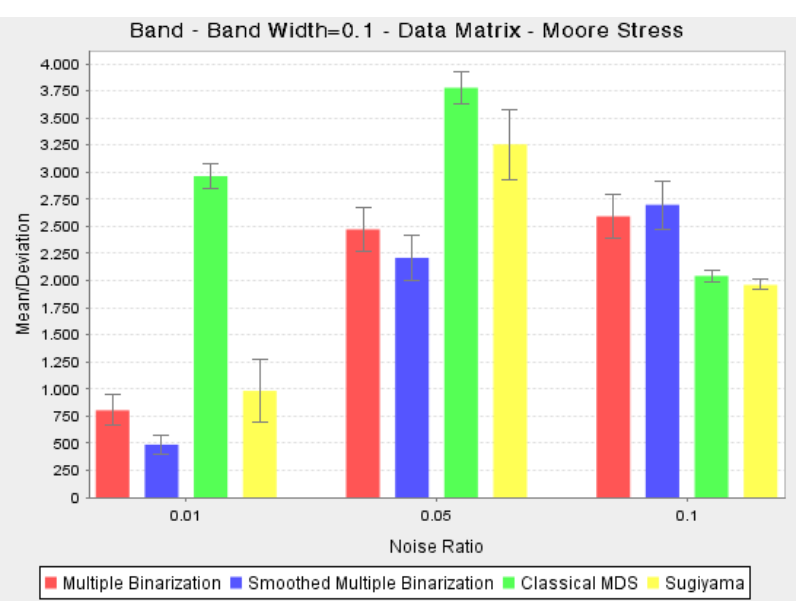

Figura 2. Gráfico do resultado da comparação dos algoritmos Sugiyama, MDS, SMB e MB utilizando a função de avaliação Moore Stress ${ }^{5}$ em uma matriz Band com largura de banda 0.1.

\section{Conclusões}

Graças à implementação da configuração do parâmetro nos padrões, foi possível analisar que os algoritmos de binarização múltipla (SMB e MB) trazem um bom resultado de ordenação, por exemplo, no padrão Band com configuração 0.1 .

Com o uso da biblioteca JFreeChart foi possível gerar gráficos automaticamente, permitindo uma comparação melhor dos algoritmos e configurações usadas nos experimentos feitos através da MRA.

\section{Agradecimentos}

Agradecimentos à FAPESP (processo no 2015/00411-6) pelo financiamento do projeto.

\footnotetext{
${ }^{1}$ Silva, C. G.; Melo, M. F.; Silva, F. P.; Meidanis, J. PQR-Sort - Using PQRTree for binary matrix reorganization. Proceedings of Journal of the Brazilian Computer Society, 2014, 1, 13.

${ }^{2}$ Wilkinson, L. The Grammar of Graphics, $2^{\text {nd }}$. Edition, 2005.

3 Medina, B.F. Reordenação de matrizes de dados quantitativos usando árvores $P Q R$. Dissertação de mestrado. Faculdade de Tecnologia, Universidade Estadual de Campinas, 2015.

${ }^{4}$ Silva, C.G.; Kawakami, W.H., Silva, M.R., Silva,C.G. Smoothed Multiple Binarization - using PQR tree, smoothing, feature vectors and thresholding for matriz reordering. Proceedings of 20th International Conference on Information Visualization, Lisboa, 2016. (A ser publicado.)

5 Niermann, S. "Optimizing the ordering of tables with evolutionary computation," The American Statistician, vol. 59, n. 1, 2005.
} 